\title{
Estrogen and raloxifene improve metaphyseal fracture healing in the early phase of osteoporosis. A new fracture-healing model at the tibia in rat
}

\author{
E. K. Stuermer $\cdot$ S. Sehmisch $\cdot$ T. Rack $\cdot$ E. Wenda • \\ D. Seidlova-Wuttke $\cdot$ M. Tezval $\cdot$ W. Wuttke . \\ K. H. Frosch $\cdot$ K. M. Stuermer
}

Received: 25 July 2008 / Accepted: 10 November 2008 /Published online: 2 December 2008

(C) The Author(s) 2008. This article is published with open access at Springerlink.com

\begin{abstract}
Background Fracture healing in osteoporosis is delayed. Quality and speed of fracture healing in osteoporotic fractures are crucial with regard to the outcome of patients. The question arises whether established antiosteoporotic drugs can further improve fracture healing.

Materials and methods Osteoporosis manifests predominantly in the metaphyseal bone. Nevertheless, an established metaphyseal fracture model is lacking. A standardized metaphyseal fracture-healing model with stable plate fixation was developed for rat tibiae. The healing process was analyzed by biomechanical, gene expression, and histomorphometric methods in ovariectomized (OVX) and sham-operated rats (SHAM), compared to standardized estrogen (E)- and raloxifene (R)-supplemented diets.

Results Estrogen and raloxifene improved the biomechanical properties of bone healing compared to OVX (Yield load: $\mathrm{SHAM}=63.1 \pm 20.8 \mathrm{~N}, \quad E=60.8 \pm 17.9 N, \quad R=44.7 \pm$ $17.5 N$, OVX $=32.5 \pm 22.0 N$ ). Estrogen vs OVX was significant based on a denser trabecular network. Raloxifene
\end{abstract}

Animal study permission from 12/5/03, Az: 509.42502/01-53.03

District Government of Braunschweig, Germany.

E. K. Stuermer $(\bowtie) \cdot S$. Sehmisch • T. Rack • E. Wenda •

M. Tezval $\cdot$ K. H. Frosch • K. M. Stuermer

Department of Trauma Surgery, Plastic and Reconstructive

Surgery, Georg-August-University of Goettingen,

Robert-Koch-Str. 40,

37099 Göttingen, Germany

e-mail: e.k.stuermer@med.uni-goettingen.de

D. Seidlova-Wuttke $\cdot$ W. Wuttke

Department of Clinical and Experimental Endocrinology,

Georg-August-University of Goettingen,

Göttingen, Germany greatly induced total callus formation $\left(R=5.3 \pm 0.9 \mathrm{~mm}^{2}\right.$, $E=4.7 \pm 0.5 \mathrm{~mm}^{2}, \quad$ SHAM $=4.51 \pm 0.61 \mathrm{~mm}^{2}, \mathrm{OVX}=$ $4.1 \pm 0.6 \mathrm{~mm}^{2}$ ), whereas estrogen mainly enhanced new endosteal bone formation. There was no correlation between the gene expression (osteocalcin, collagen $1 \alpha 1$, IGF-1, tartrate-resistant phosphatase) in the callus and the morphology and quality of callus formation.

Conclusion Raloxifene and estrogen improve fracture healing in osteoporotic bone significantly with regard to callus formation, resistance, and elasticity. The biomechanically stable metaphyseal osteotomy model with T-plate fixation presented here has proven to be appropriate to investigate fracture healing in osteoporosis.

Keywords Metaphyseal fracture healing · Osteoporosis . Raloxifene $\cdot$ Biomechanics Estrogen

\section{Introduction}

In postmenopausal osteoporosis, the increased bone turnover is accompanied by a decrease in bone mass and bone quality followed by skeletal fragility and an increased fracture risk. In the USA, the annual costs resulting from osteoporotic fractures have been estimated at $\$ 16.7$ billion [1]. In addition, according to animal experiments, osteoporotic bone shows a prolonged and impaired healing process $[2,3]$ compared with normal bone. The question arises as to whether substances, which are successful in the treatment of osteoporosis, are efficient in improving fracture healing in osteoporotic bone as well. Currently, inhibitors of bone resorption such as estrogen and raloxifene are effective in the treatment of osteoporosis in postmenopausal women [4]. They also prevent bone loss in ovariectomized rats [5, 
6]. Their effects on bone healing, particularly with regard to the healing of osteopenic metaphyseal bone, however, are still unclear.

The ovariectomized rat is a widely accepted model of osteopenia [7-9]. Bone loss in the proximal rat tibial metaphysis amounts to about $50 \%$ within 3 months after ovariectomy [8-11]. In humans, osteoporotic fractures are predominantly located at the thoracic and lumbar spine and the metaphysis of long bones, i.e., the distal radius, proximal femur, and proximal humerus. Until now, no established biomechanical stable metaphyseal fracture animal model has existed, and all studies addressing fracture healing in osteopenic bone have focused on diaphyseal fractures [5, 12-17]. But it is well known that there are important differences in fracture healing at the diaphyseal bone compared to the mechanisms of bone healing in the metaphysis. Diaphyseal bone usually heals indirectly with visible periosteal callus formation or-in absolutely stable conditions - directly with lamellar bone formation. Metaphyseal bone usually heals directly with endosteal bone bridging by micro-callus and without significant periosteal callus formation. In addition, osteoporotic changes seen in diaphyseal bone are considerably less and differ compared to those occurring in metaphyseal, trabecular bone $[6,18,19]$.

\section{Materials and methods}

Animals and operating procedure

Forty three-month old female Sprague-Dawley rats (Winkelmann, Borken, Germany) weighing 220-260 g were used for this study. Thirty rats were ovariectomized under Rompun/Ketanest $(3.5 v / v, 1 \mathrm{~mL} / \mathrm{kg})$ anesthesia. Ten rats were sham-operated for ovariectomy. During the same anesthesia, all animals also underwent the following metaphyseal osteotomy and internal plate fixation procedures for both proximal tibiae as follows.

An anterior-medial approach with an extension from the medial femur condyle to the middle of the tibia was used. A needle marked the tibial surface of the knee to obtain the correct axis for the osteotomy. The proximal tibial third was prepared in an epiperiosteal manner without harming the flexor and extensor muscles. A five-hole, $90^{\circ}$ small Tshaped titanium fixation plate XS (57-05140, Stryker Trauma, Selzach, Switzerland), which was slightly pre-bent in the transversal part, was fixed with two proximal and two distal $1.2 \mathrm{~mm}$ screws to the anterior-medial surface of the tibia. The central plate hole was left without screw. To perform the osteotomy, the plate was temporarily removed. The osteotomy was performed $7 \mathrm{~mm}$ distal to the knee surface using pulsed ultrasound (Piezosurgery ${ }^{\circledR}$, Mectron
Medical Technology, Carasco, Italy). This tool only cuts hard material and prevents muscles, tendons, nerves, and vessels from being damaged. After the osteotomy, the plate was fixed in its previous position (Fig. 1a), and an osteotomy gap of $0.5 \mathrm{~mm}$ was obtained. The muscle fascia was refixed with Vicryl ${ }^{\circledR} 4.0$ (Eticon Norderstedt, Johnson \& Johnson, Germany) after irrigation and disinfection (Braunovidon ${ }^{\circledR}$, Bayer, Leverkusen) of the operating wound. The skin was closed with brackets (Michel wound brackets $12 \times 3 \mathrm{~mm}$, Gebrueder Martin $\mathrm{GmbH} \&$ Co. KG, Tuttlingen, Germany). The animals were finally injected with a single dose of perphenacin $(5 \mathrm{mg} / \mathrm{kg}$, s.c.), followed by four injections of carprofen $(4 \mathrm{mg} / \mathrm{kg})$ for pain prophylaxis during the initial $48 \mathrm{~h}$ postoperatively.

This animal study protocol was approved by the local regional government and conformed to German animal protection laws (District Government of Braunschweig, permission from 12/5/03, Az: 509.42502/01-53.03).

Treatment groups and substances

Animals were divided into four groups:

Group I Osteopenic control, with ovariectomy (OVX) received phytoestrogen-free pelleted food (where protein supplementation was substituted with potato proteins).

Group II Sham-operated animals, furthermore referred to as "SHAM," received phytoestrogen-free pelleted food as well.
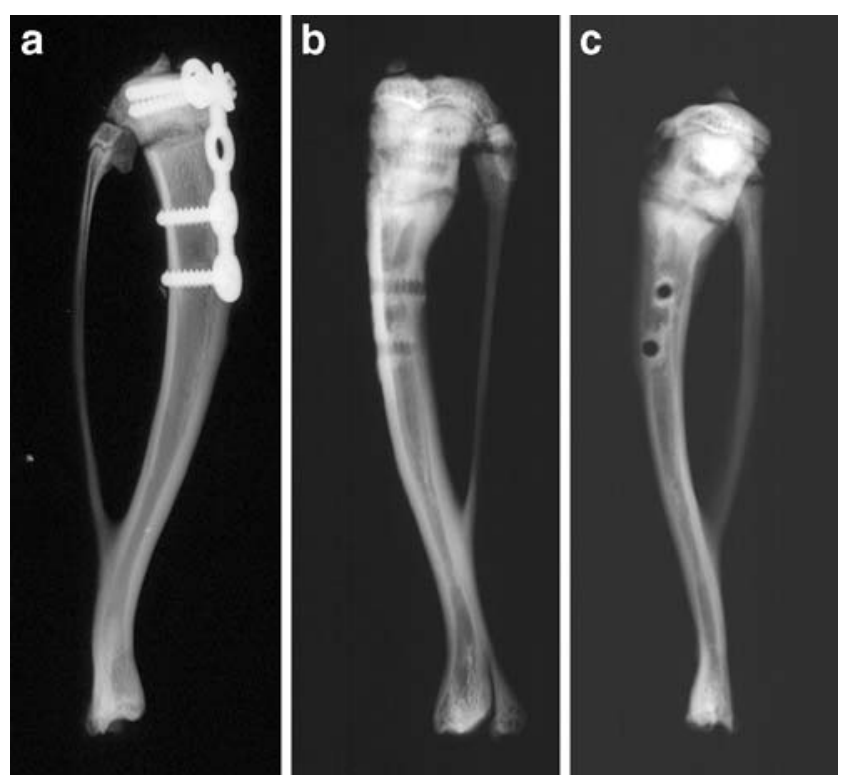

Fig. 1 X-rays of the rat tibia after the operating procedure (a), and 5 weeks after osteotomy without implant in the anterior-posterior (b) and lateral view (c). Fracture healing in progress is revealed by the vague visible fracture gap and less periosteal callus formation 
Group III Osteopenic animals received phytoestrogen-free food supplemented with estrogen in the form of estradiol benzoate (E) after ovariectomy. The average food intake per animal per day was $20 \mathrm{~g}$, so that the average $\mathrm{E}$ intake was $0.5 \mathrm{mg} /$ day.

Group IV Osteopenic animals, received phytoestrogenfree food supplemented with raloxifene (R) after ovariectomy. The average intake was $3.4 \mathrm{mg} / \mathrm{day}$ of R.

Animals were kept on these specific diets during fracture healing for a period of 5 weeks.

\section{Intravital fluorochrome labeling}

During fracture healing, the rats were subcutaneously injected with four fluorescent agents (Merck, Darmstadt, Germany) to label the process of bone formation [20]. The fluorochrome agents connect to the calcifying surfaces, which are activated just at the time of injection. They act competitively to the calcium-apatite binding. The fluorochrome labeling involved application of xylenol orange $(90 \mathrm{mg} / \mathrm{kg})$ on day 13 , calcein green $(10 \mathrm{mg} / \mathrm{kg})$ on day 18 , alizarin red $(30 \mathrm{mg} / \mathrm{kg})$ on days 24 and 26 , and tetracycline $(25 \mathrm{mg} / \mathrm{kg})$ on day 35 . The additional dose of alizarin red was given on day 26 to intensify this color. With this labeling procedure, it was possible to follow the osteoporotic fracture healing process under the influence of estrogen and raloxifene over the whole period of the trial. The chronological sequence and the quality of new built bone could be distinguished, as well as bone resorption and bone remodeling. Conclusions could be drawn regarding the individual effects of estrogen and raloxifene (periosteal, endosteal, amount of callus formation, density of callus structure) during the healing process.

\section{Endpoint and specimen preparation}

Animals were finally killed $2 \mathrm{~h}$ after tetracycline application on day 35 under anesthesia. This time was chosen because, according to our preliminary trials, fracture healing was still in progress, although not complete, and peripheral resorption of the outer callus should not occur.

Tibiae were prepared as follows: skin, muscles, and tendons were removed, and the fibula was separated at the synostosis. The plate and screws were removed (Fig. 1b,c), and the stability of the screws was observed. Tibiae were immediately stored at $-80^{\circ} \mathrm{C}$ prior to further analysis. The right and left tibiae were randomized in such a way that one was used for biomechanical testing, histological, and microradiographic analysis, and the other one was used for the analysis of the gene expression.

\section{Radiographic evaluation}

Tibiae used for morphological evaluation were thawed and continuously moistened with isotonic saline solution. Microfocus radiographs in the anterior-posterior and lateral views were taken of all tibiae in the Faxitron Cabinet X-ray System (Hewlett-Packard, $50 \mu \mathrm{m}$ x-ray beam output; model 43855A; IL 60089, USA). A highresolution film (Fuji HR-E 30 Medical X-ray) and $40 \mathrm{KV} /$ 6 min radiation were used. The description and evaluation of the fracture healing was performed in a blinded manner for all the test groups.

\section{Biomechanical testing}

Immediately after X-ray, each tibia was placed on the newly developed three-point bending and breaking test device as previously described [21]. A bending test instead of a torsional test was used because the metaphyseal tibiaunlike the metaphyseal femur or humerus - is mainly loaded in tension and compression via the tendons of the quadriceps, the biceps femoris, and the gastrocnemius muscle.

At the beginning of the test, the stamp was driven down onto the metaphyseal tibia at the point of the former osteotomy until a primary strength of $1 \mathrm{~N}$ was reached. After a final visual check of the correct tibial position, the bending test was initiated. The investigation was manually stopped when the first linear rising of the bending graph visibly decline (force between 50 to $70 \mathrm{~N}$ ) to prevent damage or fracture of the callus formation. This procedure was performed in a blinded manner with regard to the test groups.

Preparation for microscopy and microradiography

After the bending tests, the tibiae were immediately defatted in an alcohol series (not decalcified) followed by embedding in methylmethacrylate. After polymerization, $100 \pm 10 \mu \mathrm{m}$ thick longitudinal sections were cut at right angles to the plate using a specifically designed diamondcoated innerhole saw (Leica SP 1600 saw microtome, Bensheim, Germany). The saw blade had a thickness of $300 \mu \mathrm{m}$. The thickness of each slice was confirmed using a special measuring device for histological sections (Leica, Bensheim, Germany).

Microradiographies of the three central histological sections were performed on special high-resolution Kodak Professional Industrex SR45 film (100NIF) on the Faxitron Microfocus Cabinet X-ray system (Hewlett-Packard, $50 \mu \mathrm{m}$ X-ray beam output; model 43855A; IL 60089, USA) as previously described [23-26]. The bone sections were exposed for $3 \mathrm{~min}$ to $10 \mathrm{kV}$. The resolution of the images 
was approximately $0.5 \mu \mathrm{m}$ (Fig. 2a,c,e). The film was analysed by light microscopy and digital image processing (Quantimet system, Leica DM-RXE, Bensheim, Germany) to quantify the epiperiosteal and endosteal bone healing process.

After the microradiographic procedure, the three central sections were mounted on slides for histomorphologic evaluation. A light microscope (Leitz DM-RXE, Leica, Bensheim, Germany) with an I3 filter system (excitation filter 450-490 nm, diachronic mirror $510 \mathrm{~nm}$, suppression filter $515 \mathrm{~nm}$ ) was used. The best objective for evaluation of the different fluorochrome-labeled areas (Fig. 2b,d,f,h) was achieved with the transmitted and incident light objective PL Fluotar 10/0.30.
Fig. 2 Typical microradiographies and corresponding fluorochrome-labeled histological sections of tibial callus formation in the control group (a, b), as well as the sham-operated group (c, d), the raloxifene$(\mathbf{e}, \mathbf{f})$, and estrogen-treated group $(\mathbf{g}, \mathbf{h})$. Visualization of the healing process in the histological sections: unstructured new bone formation at the beginning (calcein green), first bony bridges in the osteotomy gap after 25 days (alizarin red) and considerable increase of the endosteal callus formation in the last 10 days (tetracycline yellow)
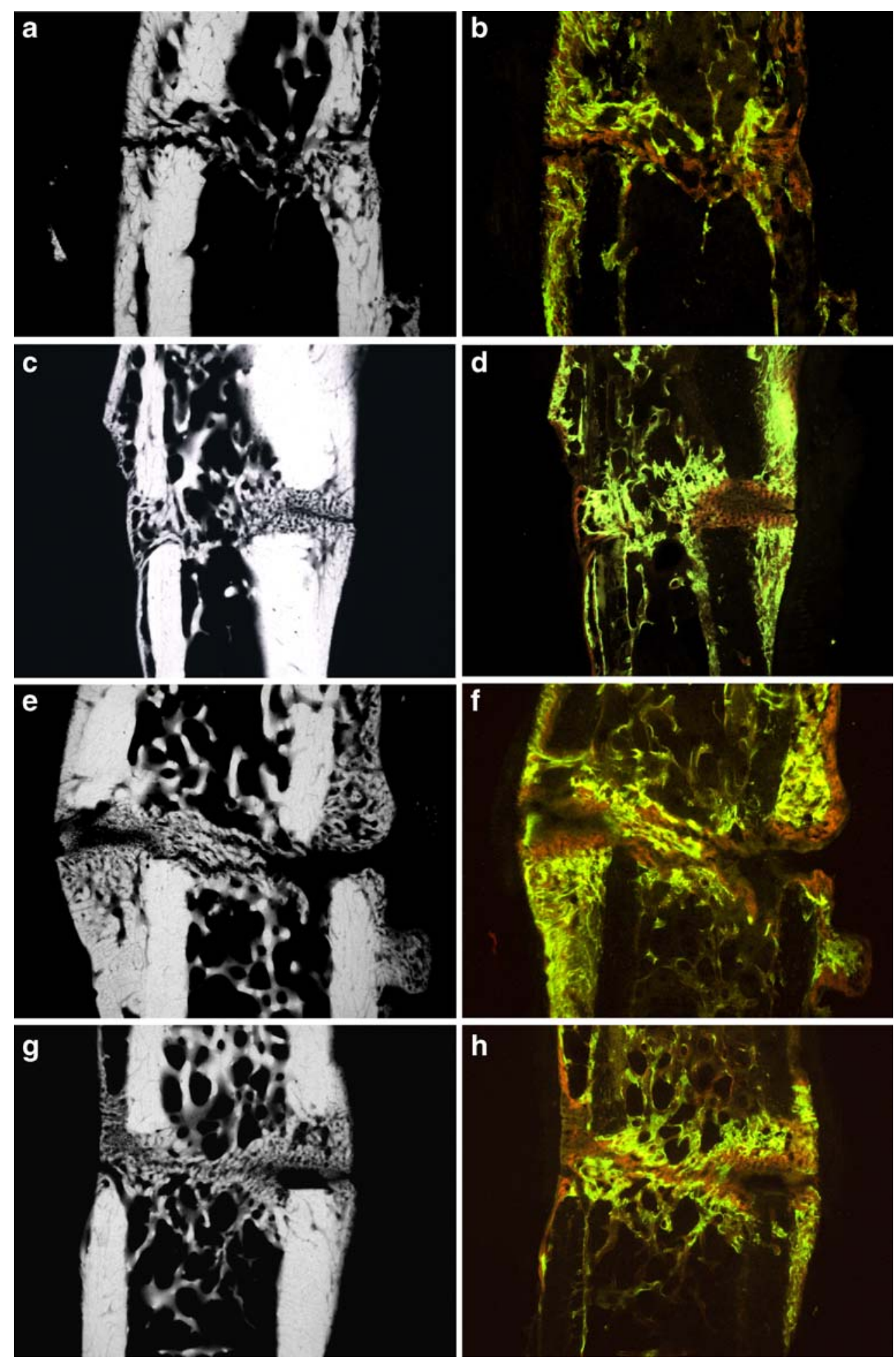
Gene analysis

Total RNA was extracted from the metaphyseal callus formation of one tibia. To determine osteocalcin (OC), insulin growth factor- 1 (IGF-1), collagen $1 \alpha 1$, and tartrateresistant phosphatase (TRAP), tissue samples were chilled in liquid nitrogen and pulverized in a 5-mL teflon container with a tissue homogenizer (Microdismembrator ${ }^{\mathrm{TM}}$, Braun, Melsungen, Germany). The extraction of RNA and measurements for real-time PCR were carried out as previously described [27]. In this study, serum analysis for estradiol$17 \beta$ was not redetermined. In preliminary studies, it has been established that, with an average $\mathrm{E}$ intake of $0.5 \mathrm{mg} /$ day, the serum concentration of estradiol-17 $\beta$ falls within the physiological range for females $(20-350 \mathrm{ng} / \mathrm{ml}$ depending on the hormonal cycle) $[21,27]$.

Evaluation and statistics

X-rays were analyzed regarding the type of fracture healing, the site and extension of callus formation, possible implant loosening, and involvement of the fibula.

On the basis of the biomechanical testing data, the stiffness and the yield load of each specimen were calculated.

The three central histological sections of each tibia and corresponding microradiographs were histomorphometrically analyzed (Leica MZ 75, Bensheim, Germany). The quantitative analysis of bone tissue was digitally determined by using the Quantimet system (Leica, Bensheim, Germany). Data were determined on histological and microradiographic images using a digital camera (Leica DC200) combined with a specific database system (Leica). For exclusively analyzing the callus formation of the metaphyseal tibia, a line was placed on the distal ends of the cortical bone of the osteotomy gap. A measuring frame centered on this line was created with a 1-mm edge length in the cranial and 1-mm edge length in the caudal direction.

For the intravitale fluorochrome-labeled histological sections, the following areas were evaluated for calcein green (CG), alizarin red, and tetracycline (yellow) labeling: periosteal callus formation dorsal, periosteal callus formation ventro-medial, and endosteal callus formation. Xylenol orange could not be evaluated because there were only small islands of xylenol orange-labeled bone surrounded by calcein green-labeled bone (Fig. 2b,d,f,h), which were visible at high resolution but not detectable by the computer system. The microradiographs of callus formation were analyzed for the following parameters: total bone area, cortical width dorsal, cortical width ventro-medial, callus width dorsal, callus width ventro-medial, callus area density, trabecular width, as well as the number of trabecular nodes and number of trabecular nodes per square millimeter.
For statistical analyses, the mean values and standard deviation for each parameter were calculated. Differences between the four tested groups were assessed using the One-way analysis of variance and the post hoc Tukey's test.

\section{Results}

Radiographic evaluation

The tibial osteotomies of all forty rats healed adequately (Fig. 1b,c) with only one infection occurring in the OVX group. In some rats, fibula fractures were detected during post mortal preparation (regardless of the treatment group), where the tibial osteotomies healed with visibly more callus formation and axial deviation was triggered by an unstable biomechanical environment and not as a result of a hormonal test factor. Therefore, these tibiae were rejected, and only eight of the OVX-group, nine SHAM-operated, seven E-, and nine R-treated rats were evaluated in this study.

The typical trabecular network of the tibial metaphysis extended from 5.5 to $9.5 \mathrm{~mm}$ below the tibia plateau in rats investigated in this study. The osteotomy level was always located in this area: In the SHAM group, it was $8.71 \pm$ $0.63 \mathrm{~mm}$ below the joint, in the OVX group $8.12 \pm 1.5 \mathrm{~mm}$, in the R group $8.43 \pm 1.05 \mathrm{~mm}$, and in the E group $8.0 \pm 0.8 \mathrm{~mm}$.

\section{Biomechanical testing}

On the basis of established strength graphs, the stiffness and yield point were calculated [21]. The yield point amounted to $32.5 \pm 22.0 \mathrm{~N}$ for the osteopenic OVX group and $44.7 \pm$ $17.5 \mathrm{~N}$ for R-treated animals, respectively. The OVX yield point was significantly lower than that for the SHAM group $(63.1 \pm 20.8 \mathrm{~N})$ and for the E group $(60.8 \pm 17.9 \mathrm{~N})$; i.e., a significant higher force was necessary for inducing irreversible plastic deformation of the callus after E supplementation. The stiffness amounted to $90.9 \pm 29.5 \mathrm{~N} / \mathrm{mm}$ in the OVX group, $82.8 \pm 43.6 \mathrm{~N} / \mathrm{mm}$ in the SHAM group, $93.5 \pm 33.3 \mathrm{~N} / \mathrm{mm}$ in the E group, and $110.3 \pm 65.2 \mathrm{~N} / \mathrm{mm}$ in the $\mathrm{R}$ group. Due to high standard deviation, however, there was no significant difference between the groups.

\section{Microradiographic evaluation}

The ventro-medial callus formation was increased after both $\mathrm{E}$ and $\mathrm{R}$ supplementation (Table 1). This increase was significant only for the latter group compared to OVX.

The trabecular width was significantly enhanced in Rtreated animals compared to OVX, as well as in E-treated animals (Fig. 3b). The number of trabecular nodes distal to the fracture callus was significantly lower in the OVX 
Table 1 Quantitative evaluation of the three central microradiographic sections of the tibial callus formation in osteopenic rats

\begin{tabular}{lcccc}
\hline & OVX & SHAM & Raloxifen & Estradiol \\
\hline Total bone area $\left(\mathrm{mm}^{2}\right)$ & $2.52 \pm 0.71$ & $2.33 \pm 0.71$ & $2.92 \pm 0.739$ & $2.64 \pm 0.24$ \\
Tibial diameter proximal $(\mathrm{mm})$ & $3.11 \pm 0.21$ & $2.76 \pm 0.26$ & $3.49 \pm 0.20$ & $2.89 \pm 0.2$ \\
Tibial diameter distal $(\mathrm{mm})$ & $2.27 \pm 0.45$ & $2.25 \pm 0.40$ & $2.67 \pm 0.35$ & $2.65 \pm 0.18$ \\
Cortical width dorsal $(\mathrm{mm})$ & $0.55 \pm 0.06$ & $0.57 \pm 0.11$ & $0.59 \pm 0.074$ & $0.56 \pm 0.17$ \\
Cortical width ventro-medial $(\mathrm{mm})$ & $0.54 \pm 0.10$ & $0.46 \pm 0.05^{*}$ & $0.51 \pm 0.065$ & $0.46 \pm 0.04^{*}$ \\
Callus width dorsal $(\mathrm{mm})$ & $0.76 \pm 0.23$ & $0.82 \pm 0.32$ & $1.04 \pm 0.449$ & $0.74 \pm 0.32$ \\
Callus width ventro-medial $(\mathrm{mm})$ & $0.41 \pm 0.13$ & $0.34 \pm 0.11$ & $0.52 \pm 0.18^{*}$ & $0.50 \pm 0.29$ \\
Callus area density $(\%)$ & $73.51 \pm 13.6$ & $80.71 \pm 7.99^{* *}$ & $75.64 \pm 13.81^{* *}$ & $7.66 \pm 1.58^{*}$ \\
Trabecular width $(\mu \mathrm{m})$ & $6.01 \pm 1.49$ & $6.28 \pm 2.3$ & 7.83 .5 & $6.03 \pm 1.67$ \\
Number of trabecular nodes & $6.71 \pm 4.83$ & $8.24 \pm 5.16^{* *}$ & $15.00 \pm 7.26^{*}$ & $19.11 \pm 14.7^{*}$ \\
Number of trabecular nodes $\left(\mathrm{mm}^{2}\right)$ & $9.55 \pm 6.84$ & $12.23 \pm 5.37^{* *}$ & $16.18 \pm 6.80^{*}$ & $21.01 \pm 11.9^{*}$ \\
\hline
\end{tabular}

Average value $+\mathrm{SD}$ of the mean

$* p<0.05$ vs $\mathrm{C} ; * * p<0.05$ vs $\mathrm{E}$ )

group than in the $\mathrm{R}$ - and E-treated groups, and the corresponding density (number of trabecular nodes per square millimeter) also followed a similar trend-trabecular density was significantly lower for OVX than for the Rand E-treated animals (Fig. 3a). There was only one significant difference between the $\mathrm{E}$ and the SHAM and R groups, respectively: the percentage of bone in the whole callus area (callus density) was significantly higher in the SHAM and R-treated animals compared to the E-treated animals.

a
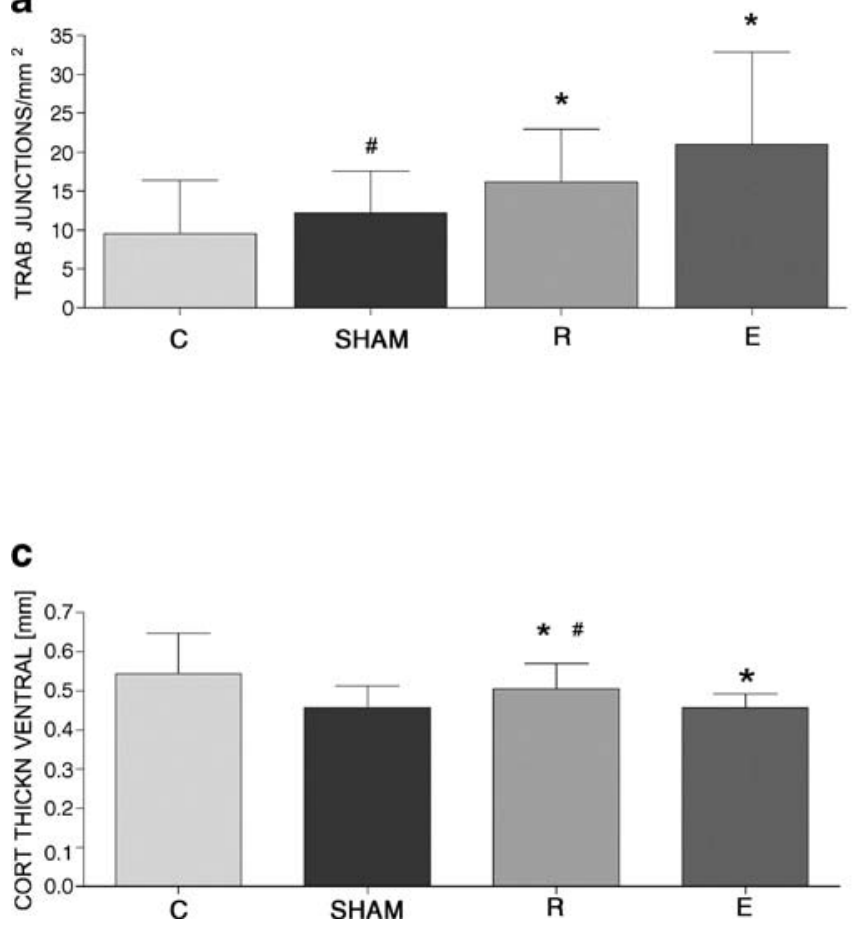

Fig. 3 Quantitative evaluation of three microradiographic sections of each tibia callus regarding the trabecular $(\mathbf{a}, \mathbf{b})$ and cortical bone $(\mathbf{c}, \mathbf{d})$ in the osteoporotic control group $(C)$, in the sham-operated group, and
The ventro-medial cortical bone was significantly thicker for OVX animals compared to the SHAM and the E group.

Evaluation of the intravitale fluorochrome labeling

$\mathrm{R}$ induced the most callus formation (Fig. 4a), as well as significantly improved fracture healing at ventro-medial, dorsal, and endosteal locations (as determined by fluorochrome labeling) at different times compared to the OVX group (Table 2). R nearly doubled the callus amount at each

b

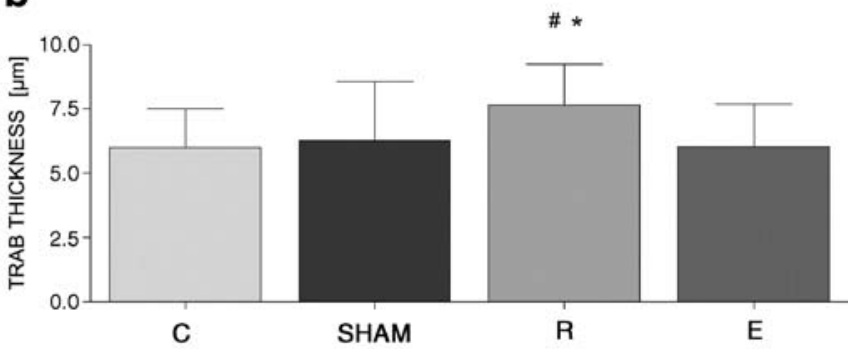

d

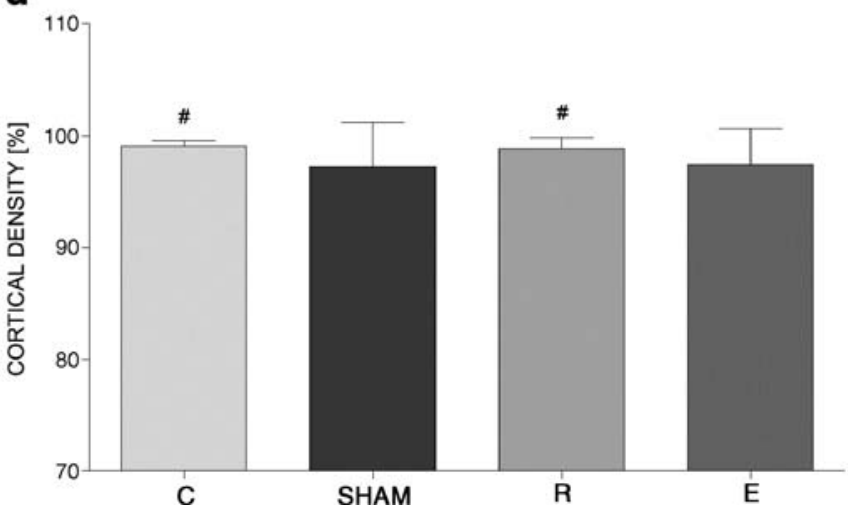

after supplementation with raloxifene $(R)$ or estrogen $(E)$. Values are expressed as the mean $\pm \mathrm{SD}$ (asterisk $p<0.01$ vs $\mathrm{C}$, pound sign $p<0.01$ vs E) 
Fig. 4 Quantitative evaluation of the fracture healing process in fluorochrome-labeled histological sections of the tibial callus ( $C G$ calcein green, $A K$ alizarin red, $T C$ tetracycline). Total callus formation (a) consisting of periosteal callus dorsal, periosteal callus ventromedial, and endosteal callus formation. Differentiation of the total callus formation in three regions of interest: the endosteal region (b), the ventro-medial region (c), and the dorsal region (d). Values are expressed as the mean $\pm \mathrm{SD}\left({ }^{*} p<0.01\right.$ vs $\left.\mathrm{C}\right)$ a

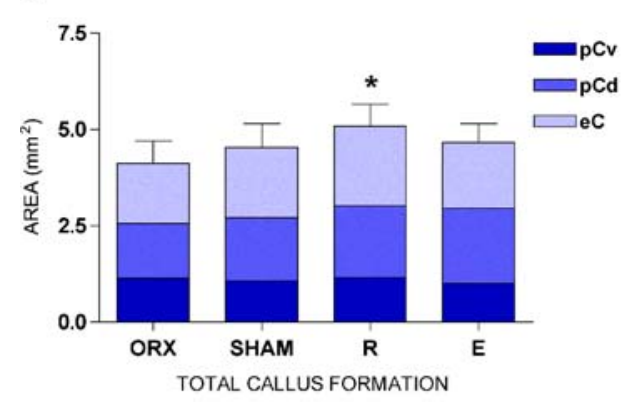

C

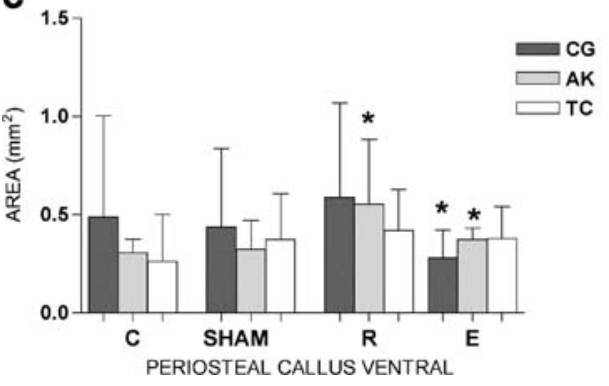

b

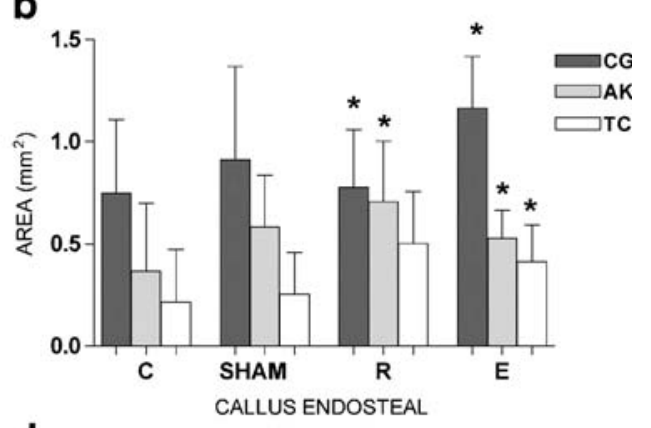

d

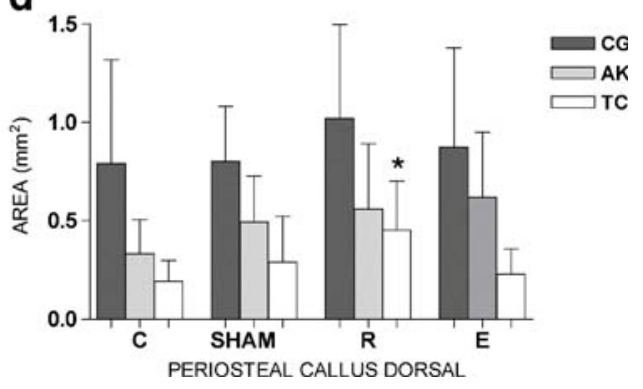

time of labeling with exception of the CG period at the endosteal callus. For E-treated rats, only the area of endosteal callus formation showed a significant increase (Fig. 4b), which proves higher bone formation at all times of the monitored healing process. In the initial 3 weeks, the ventro-medial callus formation (Fig. 4c) was significantly smaller in the E group compared to OVX, but the E group made up ground in the following 2 weeks. Etreated rats developed the same level of total callus-like SHAM-operated rats, and the distribution between ventromedial, dorsal, and endosteal locations was very similar as well.
Gene analysis

After killing the animals, gene expression of OC, IGF-1, collagen $1 \alpha 1$, and TRAP was determined in the callus formation of one tibia from each rat. Based on the ovariectomy (set to $100 \%$ ), gene expression of OC-a typical osteoblast marker-was significantly reduced in E-treated animals $(54.9 \pm 34.1 \%)$ and slightly enhanced at SHAM $(107.5 \pm 34.5 \%)$ and under R treatment $(111.1 \pm 79.3 \%)$. For the gene expression of IGF-1 and collagen $1 \alpha 1$ - two other osteoblast marker genes - a downregulation of both compared to OVX was observed after supplementation with E (61.2 \pm

Table 2 Quantitative analysis of the process of bone healing at the metaphyseal tibia after intravitale fluorochrome labeling with calcein green (CG) (day 18), alizarin red (AK) (day 24 and 26), and tetracycline (TC) (day 35)

\begin{tabular}{|c|c|c|c|c|}
\hline & osteopenic C & SHAM & RALOXIFEN & ESTRADIOL \\
\hline Total callus ventro-medial $\left(\mathrm{mm}^{2}\right)$ & $1.14 \pm 0.58$ & $1.07 \pm 0.40$ & $1.42 \pm 1.03$ & $1.00 \pm 0.27$ \\
\hline Callus area ventro-medial CG & $0.49 \pm 0.51$ & $0.44 \pm 0.40$ & $0.59 \pm 0.48$ & $0.28 \pm 0.14^{*}$ \\
\hline Callus area ventro-medial AK & $0.31 \pm 0.07$ & $0.32 \pm 0.15$ & $0.55 \pm 0.33^{*}, * *$ & $0.37 \pm 0.06^{*}$ \\
\hline Callus area ventro-medial TC & $0.26 \pm 0.24$ & $0.37 \pm 0.23$ & $0.42 \pm 0.21$ & $0.38 \pm 0.16$ \\
\hline Total callus dorsal $\left(\mathrm{mm}^{2}\right)$ & $1.42 \pm 0.80$ & $1.62 \pm 0.60$ & $1.86 \pm 0.95$ & $1.96 \pm 0.59$ \\
\hline Callus area dorsal CG & $0.79 \pm 0.53$ & $0.80 \pm 0.28$ & $1.02 \pm 0.48$ & $0.87 \pm 0.51$ \\
\hline Callus area dorsal $\mathrm{AK}$ & $0.33 \pm 0.17$ & $0.50 \pm 0.23$ & $0.56 \pm 0.33$ & $0.62 \pm 0.33$ \\
\hline Callus area dorsal TC & $0.19 \pm 0.11$ & $0.29 \pm 0.23$ & $0.45 \pm 0.25^{*}$ & $0.23 \pm 0.13$ \\
\hline Total callus endosteal $\left(\mathrm{mm}^{2}\right)$ & $1.56 \pm 0.59$ & $1.82 \pm 0.62$ & $2.06 \pm 0.58^{*}$ & $1.71 \pm 0.49$ \\
\hline Callus area endosteal CG & $0.75 \pm 0.36$ & $0.91 \pm 0.46$ & $0.78 \pm 0.28$ & $1.16 \pm 0.25^{*}$ \\
\hline Callus area endosteal $\mathrm{AK}$ & $0.37 \pm 0.33$ & $0.58 \pm 0.25^{*}$ & $0.71 \pm 0.29 *$ & $0.53 \pm 0.14^{*}$ \\
\hline Callus area endosteal TC & $0.21 \pm 0.26$ & $0.26 \pm 0.20$ & $0.50 \pm 0.25^{*}$ & $0.41 \pm 0.18^{*}$ \\
\hline Total callus area $\left(\mathrm{mm}^{2}\right)$ & $4.13 \pm 0.56$ & $4.51 \pm 0.61$ & $5.34 \pm 0.65^{*}$ & $4.66 \pm 0.45$ \\
\hline
\end{tabular}

Average value $+\mathrm{SD}$ of the mean

${ }^{*} p<0.05$ vs $\mathrm{C} ;{ }^{* *} p<0.05$ vs SHAM) 
$34.6 \%$ and $68.7 \pm 42.9 \%$, respectively). A reduced expression of IGF-1 $(90.5 \pm 39.9 \%)$ and collagen- $1 \alpha 1(88.3 \pm 45.9 \%)$ was also seen for R-treated compared to OVX animals, but these decreases were not significant. SHAM-operated animals showed the same level of IGF-1 $(99.9 \pm 60.6 \%)$ and collagen- $1 \alpha 1(101.9 \pm 48.1 \%)$ as the OVX group. The expression of the TRAP gene - an osteoclast marker - was nearly at the same level in SHAM $(105.2 \pm 88.6)$ as in OVX. It was lower for R-treated animals $(76.4 \pm 33.1 \%)$ but not significantly reduced when compared to the E-treated group $(82.3 \pm 54.2 \%)$.

\section{Discussion}

Osteoporotic fractures are still an unsolved problem for the surgeon as well as for the patient. There are two ways of improving the fracture healing process: first, the developments of special implants to avoid implant failure; second, the improvement of bone quality to speed up and improve callus formation and otherwise to biologically advance implant fixation. This study focused on the second option. The newly developed and biomechanical stable metaphyseal osteotomy model with mini-plate osteosynthesis in the osteopenia model rat provides the opportunity to test various substances regarding their influence on fracture healing in the osteoporotic bone.

\section{Metaphyseal osteotomy and osteosynthesis model}

Former studies of fracture healing in osteoporosis in rats were essentially performed at the diaphyseal tibia [13] or femur $[5,14,28]$. Studies which address the metaphyseal bone healing were all performed in healthy as opposed to osteoporotic bone. They were performed in rat as a pullout trial [29], in sheep as a partial osteotomy [30], or in rabbits as defect healing [31] or under varying biomechanical stability [32]. The challenge of the model described in here is the biomechanically stable fixation of bone after onset of the metaphyseal tibia fracture. With the five-hole small T-plate and fixation with mildly converging screws in the epiphyseal tibia, the problem could be solved.

However, the biomechanical stability is dependent on the integrity of the fibula. If the stabilizing column of the fibula failed, the metaphyseal fracture healed in a different manner, with instability and with another kind of callus formation, although no non-union occurred. It must be assumed that this kind of unstable fracture would not heal in humans without further problems arising. Therefore in this study, some tibiae were excluded, though they were healed because of their different biomechanical starting position. It can be assumed that the biomechanical instability influences fracture healing to a great extent and interfere with the effects of the antiosteoporotic drugs, estrogen and raloxifene, so that the overall effect of these two substances may be masked by the biomechanical stimulation. The high biomechanical stability of this model in general (with intact fibula) is visible in the endosteal healing associated with slight periosteal callus formation as well. This is typical for fracture healing under stable conditions at the metaphyseal site. In case of instability, periosteal callus formation is notably accelerated as described by Claes et al. [33, 34].

A fracture-healing model in osteoporosis represented at the diaphyseal femur or tibia of the rat is not adequate for two reasons. Firstly, the mechanisms of metaphyseal fracture healing are completely different from that seen in diaphyseal bone. Diaphyseal bone essentially heals with periosteal callus formation, while metaphyseal bone usually heals endosteally with micro-callus and with only gentle periosteal callus formation. In the presented animal model, a small periosteal callus formation could be evaluated because the chosen bridge plating osteosynthesis and the $0.5 \mathrm{~mm}$ osteotomy gap allow micromotions, which triggered minimal periosteal callus. The second reason is that osteoporotic fractures in humans occur typically in the metaphysis, as osteoporosis affects the metaphyseal bone to a larger degree and in another manner than the diaphyseal bone. After 3-month duration, rats lose up to $50 \%$ bone mineral density after ovariectomy in the metaphyseal tibia [10], much more than in diaphyseal bone [35]. The trabecular structure degrades in the metaphyseal bone, while the diameter of the diaphysis enlarges to compensate for the thinning of cortical bone. This results in an extensive degradation of the metaphyseal bone, whereas the diaphyseal bone is comparatively less affected. The loss in diaphyseal biomechanical stability is then often not as high as expected [6, 19, 20, 36].

The newly developed metaphyseal fracture rat model was created with a standardized $0.5 \mathrm{~mm}$ fracture gap. This fracture gap was chosen because metaphysis fracture healing occurs with less periosteal callus formation than in the diaphysis. To evaluate the potency of a substance in stimulating fracture healing (at the morphological level), it is necessary to have a defined area for the evaluation: the $0.5 \mathrm{~mm}$ fracture gap represents this area. It is well known that small fracture gaps $(<2 \mathrm{~mm})$ also ensure better progression of fracture healing, whereas wider gaps are more likely to interfere in this process $[33,34]$. The duration of 5 weeks after performing the osteotomy was chosen because fracture healing was, according to our preliminary trials, still in progress and not yet completed [35].

Influence of estrogen and raloxifene on fracture healing

The Food and Drug Administration has approved the rat as an osteopenia model in research; animals develop substantial osteoporosis within a few weeks. In this study, the 
mature model of osteoporosis was used. Fracture healing at the metaphyseal tibia was improved by estrogen and raloxifene. These findings are similar to those observed by other groups who studied diaphyseal bone $[5,15,37,38$, 39]. In the diaphyseal bone, there was no insight regarding trabecular structure and its remodeling. Both substances were used in established concentration of oral supplementation $[5,21,22,24,39]$. The sufficiency of oral administration of $\mathrm{E}$ was proved by the measured quality and quantity of the fracture callus: Most values were even similar to those found at SHAM-operated rats which had no ovariectomy. The presented metaphyseal fracture-healing model was able to differentiate the individual effects on the fracture healing process of these two medicaments of first choice in osteoporosis.

In postmenopausal osteoporosis, the activity of osteoblasts and osteoclasts is very high due to a lack of ovarian hormones, especially of estrogen; this process is called high-turnover osteoporosis. The action of $\mathrm{E}$ is essential for maintenance of normal bone structure and thereby of bone strength [40, 41]. Interestingly the values of OC, IGF- 1 , collagen $1 \alpha 1$, and TRAP in the callus formation did not differ significantly between OVX and SHAM, although there was a lack of estrogen in OVX. A significant downregulation of all measured genes was detected for the E-treated group compared to OVX and SHAM, which means that osteoblast and osteoclast activity after E supplementation was suppressed. In contrast to what had been expected, it was not suppressed in SHAM. Raloxifene as a selective estrogen receptor modulator (SERM) lies in between the other two extreme values. Including callus morphology and quality, there seemed to be no correlation between the gene expression of OC, IGF-1, collagen $1 \alpha 1$, and TRAP and the metaphyseal fracture healing: both "positive control groups," SHAM and E, had an equal extent of callus formation and callus quality but a significantly different level of gene expression. In similarity with previous studies, gene expression in the callus formation is highly variable [42-44].

The occurrence of increased callus formation, as found for raloxifene, does not inevitably imply better mechanical quality. For estrogen-treated animals and SHAM, the yield point was significantly higher than in the OVX control group, which suggests an earlier occurrence of plastic deformation resulting in micro fracturing in the osteopenic animals; callus formation is less elastic and therefore less resistant to interfering forces. In terms of the stiffness, raloxifene showed a smaller improvement than estrogen, which had a significant higher value than the OVX group.

\section{Conclusion}

Estrogen and raloxifene improve fracture healing in osteoporotic bone after osteotomy and stable internal fixation. The quality of the endosteal and periosteal healing process resembles that of healthy bone, represented by animals with intact ovaries (SHAM).

The metaphyseal tibial fracture model presented here has proven to be suitable for the evaluation of different agents used in the treatment of osteoporosis. In the ovariectomized rat, osteopenia at the metaphyseal tibia is highly comparable to the disease that manifests in humans with osteoporotic fractures of the distal radius, the hip, or the proximal humerus. However, this animal model provides the opportunity of testing calcium, vitamins, hormones, phytoestrogenes, SERM, and bisphosphonates and observing the potential abilities of these compounds in improving fracture healing in this important bone disease.

Conflict of interest statement All authors have no conflict of interest.

Open Access This article is distributed under the terms of the Creative Commons Attribution Noncommercial License which permits any noncommercial use, distribution, and reproduction in any medium, provided the original author(s) and source are credited.

\section{References}

1. Ray NF, Chan JK, Thamer M, Melton LJ (1997) III. Medical expenditures for the treatment of osteopenic fracture in the United States in 1995: Report from the National Osteoporosis Foundation. J Bone Miner Res 12:24-35. doi:10.1359/jbmr.1997.12.1.24

2. McCann RM, Colleary G, Geddis C, Clarke SA, Jordan GR, Dickson GR, Marsh D (2008) Effect of osteoporosis on bone mineral density and fracture repair in a rat femoral fracture model. J Orthop Res 26:384-393. doi:10.1002/jor.20505

3. Hao YL, Zhang G, Wang YS, Qin L, Hung WY, Leung K, Pei FX (2007) Changes of microstructure and mineralized tissue in the middle and late phase of osteoporotic fracture healing in rats. Bone 41:631-638. doi:10.1016/j.bone.2007.06.006

4. Rodan GA, Martin TJ (2000) Therapeutic approaches to bone disease. Science 289:1508-1514. doi:10.1126/science. 289.5484.1508

5. Cao Y, Mori S, Mashiba T, Westmore MS, Ma L, Sato M, Akiyama T, Shi L, Komatsubara S, Miyamoto K, Norimatsu H (2002) Raloxifene, estrogen and alendronate affect the processes of fracture repair differently in ovariectomized rats. J Bone Miner Res 17:2237-2246. doi:10.1359/jbmr.2002.17.12.2237

6. Danielsen CC, Mosekilde L, Svenstrup B (1993) Cortical bone mass, composition, and mechanical properties in female rats in relation to age, long-term ovariectomy, and estrogen substitution. Calcif Tissue Int 52:26-33. doi:10.1007/BF00675623

7. Li XJ, Jee WSS, Ke HZ, Mori S, Akamine T (1991) Age related changes of cancellous and cortical bone histomorphometry in female Sprague-Dawley rats. Cells Mater Suppl 1:25-35

8. Wronski TJ, Yen CF (1991) The ovariectomized rat as an animal model for postmenopausal bone loss. Cells Mater Suppl 1:69-74

9. Frost HM, Jee WSS (1992) On the rat model of human osteopenias and osteoporosis. Bone Miner 18:227-236. doi:10.1016/0169-6009(92)90809-R 
10. Wronski TJ, Lowry PL, Walsh CC, Ignaszewski LA (1985) Skeletal alterations in ovariectomized rats. Calcif Tissue Int 37:324-328. doi:10.1007/BF02554882

11. Faugerer MC, Okamoto S, DeLuca HF, Malluch HH (1986) Calcitriol corrects bone loss induced by oophorectomy in rats. Am J Physiol 250:E35-E38

12. Schmidmaier G, Wildemann B, Bail H, Lucke M, Stemberger A, Flyvbjerg A, Haas NP, Raschke M (2001) Local application of growth factors (insulin-like growth factor-1 and Transforming growth factor- $\beta$ ) from a biodegradable poly(D,L-lactide) coating of osteosynthetic implants accelerated fracture healing in rats. Bone 28:341-350. doi:10.1016/S8756-3282(00)00456-7

13. Nakajima A, Shimoji N, Shiomi K, Shimizu S, Moriya H, Einhorn TA, Yamazaki M (2002) Mechanisms for the enhancement of fracture healing in rats treated with intermittent low-dose human parathyroid hormone (1-34). J Bone Miner Res 17:2038-2047. doi:10.1359/jbmr.2002.17.11.2038

14. Kubo T, Shiga T, Hashimoto J, Yoshioka M, Honjo H, Urabe M, Kitajima I, Semba I, Hirasawa Y (1999) Osteoporosis influences the late period of fracture healing in a rat model prepared by ovariectomy and low calcium diet. J Steroid Biochem Mol Biol 68:197-202. doi:10.1016/S0960-0760(99)00032-1

15. Li C, Mori S, Li J, Kaji Y, Akiyama T, Kawanishi J, Norimatsu H (2001) Long-term effect of incadronate disodium (YM-175) on fracture healing of femoral shaft in growing rats. J Bone Miner Res 16:429-436. doi:10.1359/jbmr.2001.16.3.429

16. Li J, Mori S, Kaji Y, Akiyama T, Norimatsu H (2000) Concentration of bisphosphonate (incadronate) in callus area and is effects on fracture healing in rats. J Bone Miner Res 15:20422051. doi:10.1359/jbmr.2000.15.10.2042

17. Hatano H, Siegel HJ, Yamagiwa H, Bronk JT, Turner RT, Bolander ME, Sarkar G (2004) Identification of estrogen-related genes during fracture healing, using DANN microarray. J Bone Miner Metab 22:224-235. doi:10.1007/s00774-003-0482-y

18. Peng Z, Tuukkanen J, Zhang H, Jämsä T, Väänänen HK (1994) The mechanical strength of bone in different rat models of experimental osteoporosis. Bone 15:523-532. doi:10.1016/8756-3282(94)90276-3

19. Aerssens J, van Audekercke R, Talalaj M, Geusens P, Bramm E, Dequeker J (1996) Effect of $1 \alpha$-Vitamin D3 and Estrogen therapy on cortical bone mechanical properties in the ovariectomized rat model. Endocrinology 137:1358-1364. doi:10.1210/en.137.4.1358

20. Rahn BA (1976) The fluorochrome sequence labelling of the bone. Nova Acta Leopold 44:249-255

21. Stuermer EK, Seidlova-Wuttke D, Sehmisch S, Rack T, Wille J, Frosch KH, Wuttke W, Stuermer KM (2006) Standardized bending and breaking test for the normal and osteoporotic metaphyseal tibias of the rat: effect of estradiol, testosterone, and raloxifene. $\mathrm{J}$ Bone Miner Res 21:89-96. doi:10.1359/JBMR.050913

22. Schenk RK (1965) The histological preparation of undecalcified bone. Acta Anat (Basel) 60:3-19. doi:10.1159/000142633

23. Stuermer EK, Sehmisch S, Rack T, Boeckhoff J, Wuttke W, Seidlova-Wuttke D, Stuermer KM (2008) Details of estrogen, testosterone and raloxifene influence on the microstructure and biomechanics of the osteoporotic bone. World J Urol (accepted)

24. Stuermer KM (1980) The microradiography of the bone. Technique, expressiveness and planimetry. Unfallheilk $\mathrm{Hft}$ 148:247-251

25. Frost HM (1959) Staining of fresh, undecalcified, thin bone section. Stain Technol 33:135-146

26. Walsh WR, Sherman P, Howlett CR, Sonnabend DH, Ehrlich MG (1997) Fracture healing in a rat osteopenia model. Clin Orthop Relat Res 342:218-227. doi:10.1097/00003086-199709000-00029

27. Seidlova-Wuttke D, Stuermer KM, Stuermer EK, Sehmisch S, Wuttke W (2006) Contrasting effects of estradiol, testosterone and of a black cohosh extract on density, mechanical properties and expression of several genes in the metaphysis of the tibia and on fat tissue of orchidectomized rats. Maturitas 55S:64-74. doi:10.1016/j.maturitas.2006.06.018

28. Claes LE, Heigele CA, Neidlinger-Wilke C, Kaspar D, Seidl W, Margevicius KJ, Augat P (1998) Effects of mechanical factors on the fracture healing process. Clin Orthop Relat Res 355 (Suppl):132-147. doi:10.1097/00003086-199810001-00015

29. Aspenberg P, Wermelin K, Tengwall P, Fahlgren A (2008) Additive effects of PTH and bisphoshonates on the bone healing response to metaphyseal implants in rats. Acta Orthop 79:111115. doi:10.1080/17453670710014851

30. Claes L, Veeser A, Göckelmann M, Simon U, Ignatius A (2008) A novel model to study metaphyseal bone healing under defined biomechanical conditions. Arch Orthop Trauma Surg 25 (Epub ahead of print)

31. Morgan EF, Mason ZD, Bishop G, Davis AD, Wigner NA, Gerstenfeld LC, Einhorn TA (2008) Combined effects of recombinant human BMP-7 (rhBMP-7) and parathyroid hormone (1-34) in metaphyseal bone healing. Bone (Epub ahead of print)

32. Tsiridis E, Morgan EF, Bancroft JM, Song M, Kain M, Gerstenfeld L, Einhorn TA, Bouxsein ML, Tornetta P 3rd (2007) Effects of OP1 and PTH in a new experimental model for the study of metaphyseal bone healing. J Orthop Res 25:1193-1203

33. Claes L, Augat P, Suger G, Wilke HJ (1997) Influence of size and stability of the osteotomy gap on the success of fracture healing. J Orthop Res 15:577-584. doi:10.1002/jor.1100150414

34. Iwaniec UT, Moore K, Rivera MF, Myers SE, Vanegas SM, Wronski TJ (2007) A comparative study of the bone-restorative efficacy of anabolic agents in aged ovariectomized rats. Osteoporos Int 18:351-362. doi:10.1007/s00198-006-0240-9

35. Blythe JG, Buchsbaum HJ (1976) Fracture healing in estrogentreated and castrated rats. Obstet Gynecol 48:351-352

36. Ohishi T, Takahashi M, Kushida K, Hoshio H, Tsuchikawa T, Naitoh K (1998) Changes of biochemical markers during fracture healing. Arch Orthop Trauma Surg 118:126-130. doi:10.1007/ s004020050331

37. Namkung-Matthai H, Appleyard R, Jansen J, Hao Lin J, Maastricht S, Swain M, Mason RS, Murrell GA, Diwan AD, Diamond T (2000) Osteoporosis influences the early period of fracture healing in a rat osteoporotic model. Bone 28:80-86. doi:10.1016/S8756-3282(00)00414-2

38. Miwa M, Sibonga JD, Sarkar G, Bronk JT, Mizuno K, Turner RT, Bolander ME (1999) Estrogen deficiency causes delayed fracture repair. Trans Orthop Res Soc 24:482

39. Bolander ME, Sabbagh R, Jeng C, Vivianno D, Boden SD (1992) Estrogen treatment during fracture repair strengthens healing callus in an osteoporotic model. Trans Orthop Res Soc 17:138

40. Klein P, Bail HJ, Schell H, Michel R, Amthauer H, Bragulla H (2004) Are bone turnover markers capable of predicting callus consolidation during bone healing? Calcif Tissue Int 75:40-49. doi:10.1007/s00223-004-0183-0

41. Seebeck P, Bail HJ, Exner C, Schell H, Michel R, Amthauer H, Bragulla H, Duda GN (2005) Do serological tissue turnover markers represent callus formation during fracture healing? Bone 37:669-677. doi:10.1016/j.bone.2005.06.008

42. Kalu DN (1991) The ovariectomized rat model of postmenopausal bone loss. Bone Miner 15:175-191. doi:10.1016/0169-6009(91)90124-I

43. Wronski TJ, Dann LM, Horner SL (1989) Time course of vertebral osteopenia in ovariectomized rats. Bone 10:295-301. doi:10.1016/8756-3282(89)90067-7

44. Brand RA, Rubin CT (1987) Fracture healing. In: Albright JA, Brand RA (eds) The scientific basis of orthopaedics. Appleton \& Lange, Norwalk/Connecticut, pp 325-340 